\title{
The effect of water on the solid state characteristics of pharmaceutical excipients: Molecular mechanisms, measurement techniques, and quality aspects of final dosage form
}

\author{
Gergely Szakonyi, Romána Zelkó \\ University Pharmacy Department of Pharmacy Administration, Semmelweis University, Budapest, Hungary
}

\begin{abstract}
In this paper we give an overview about the interaction of water molecules with pharmaceutical excipients. Most of these excipients are amorphous or partially amorphous polymers and their characteristics are very sensitive to the water content. In the course of the manufacturing processes water sorption is possible, therefore in some cases it is important to strictly control the residual moisture content of a dosage form. There are several mechanisms of water sorption, like water is able to bind to polar groups of hygroscopic excipients and could also exist in the capillary system of amorphous excipients. Several techniques are available to characterise the states of water inside the materials and the effects of residual water on polymers. For this purpose water sorption measurements, differential scanning calorimetry and the Fourier-transform infrared spectroscopy are reviewed. The importance of water content and storage conditions of pharmaceuticals on the properties of the final dosage forms are also demonstrated with practical examples.
\end{abstract}

Key words: Differential scanning calorimetry, free volume, FT-IR spectroscopy, polymer, positron annihilation lifetime spectroscopy, water sorption

\section{INTRODUCTION}

Water can be found everywhere in nature in each three states (solid, liquid, and gaseous). Liquid water is participant in many different manufacturing processes including pharmaceutical processes. Base of liquid dosage forms is almost exclusively water, but it can be found in half-solid dosage forms, such as hydrophilic ointments, as well. In the case of solid dosage forms usually it is essential to keep the water content low, because it could harmfully affect the physico-chemical, chemical, and microbiological stability of product. However, manufacturing processes often involve the use of water (e.g., wet granulation,

\section{Address for correspondence:}

Prof. Romána Zelkó,

University Pharmacy Department of Pharmacy Administration, Semmelweis University, Hőgyes E Street 7-9, Budapest, Hungary.

E-mail: zelrom@gytk.sote.hu

\begin{tabular}{|l|l|}
\hline \multicolumn{2}{|c|}{ Access this article online } \\
\hline Quick Response Code: & Website: \\
\hline & www.jpionline.org \\
\cline { 2 - 3 } & DOI: \\
\hline
\end{tabular}

spray-drying, coating processes, etc.), but most of them have to be removed in later steps of manufacturing.

Most common dosage form is the tablet, where largest part of the total weight is frequently composed of different excipients. Therefore, it is of impact to analyse the interaction of water molecules with excipients and the possible outcomes of these interactions.

Pharmaceutical excipients can be classified into two large categories based on their molecular order. The main categories are the crystalline, the amorphous and their mixture, the semicrystalline or partially amorphous state. The amorphous state has gained considerable attention in pharmaceutical industry because in this state the material is thermodynamically unstable, and characterised by its unique physico-chemical features reviewed by several articles. ${ }^{[1-4]}$ Unlike to crystalline solids, an amorphous solid may have only a short-range molecular order, and it has no long-range order of molecular packing like in the crystalline state. ${ }^{[3]}$ Since the molecules or clusters of molecules are randomly arranged, they occupy the volume in larger extent than that of the crystalline state having the same chemical composition. The excess volume (the so-called free volume) that is available for rotational and translational motions can be considered as microvoids inside the polymer domain. ${ }^{[1]}$ 
One of the most important parameter of an amorphous material is its glass transition temperature $\left(\mathrm{T}_{\mathrm{g}}\right)$. Under the glass transition temperature an amorphous solid is in the glassy state where rotational or translational motions of the molecules are restricted and only vibration is possible. ${ }^{[5]}$ By heating the solid over its $\mathrm{T}_{\mathrm{g}}$ value large scale molecular movements are allowed and the glassy-to-rubbery transition occurs. In this state the material has liquid-like structural characteristics but of very high viscosity. ${ }^{[2]}$

The amorphous state commonly occurs with high molecular weight pharmaceutical excipients and large peptides and proteins, but it can be found in the case of small organic or inorganic molecules, as well. ${ }^{[2]}$ However, in the latter case crystallization can easily take place due to the heating of the system or water vapour absorption. ${ }^{[6]}$ Amorphous solids are in not-equilibrium state therefore some pharmaceutical excipients have the property to exist persistently in amorphous form, while others require careful usage to prevent crystalline transition. ${ }^{[3]}$

In the light of excipient-water interactions amorphous or partially amorphous hydrophilic polymers are the most important materials. In fact, Hodge et al. investigated the semi crystalline poly (vinyl alcohol) samples and showed that water first encountered with the amorphous region of the material and the crystalline region was only affected by the amorphous/ crystalline interface if excess water was available. ${ }^{[7]}$ In contrast to the hydrophilic polymers, hydrophobic polymers sorb only low amount of moisture from the environment therefore serious changes cannot be expected. ${ }^{[8]}$

The importance of amorphous state in the case of water vapour sorption can be pointed out by the example of various sugarbased materials that are widely used in the pharmaceutical industry. Crystalline sugars can interact with water vapour by adsorption and deliquescence mechanisms, because the predominant interaction in this case is the surface adsorption by weak interaction forces. If the relative humidity reaches a critical value one can experience the phenomenon of deliquescence when sugar molecules start to dissolve at the surface. On the other hand amorphous sugars have a tendency to absorb large amount of water molecules. This is due to the fact that water molecules are absorbed into the bulk structure of amorphous materials, rather than being restricted on the surface of individual particles. This water absorption is the cause of the marked changes in the physicochemical properties of amorphous sugars, since in this case every sugar molecule is affected by interaction with water molecules. ${ }^{[9]}$

A number of excipients used in the pharmaceutical industry are amorphous or partially amorphous hydrophilic polymers. These materials owing to its polar functional groups are able to sorb (adsorb and absorb) large amount of water that can affect their functionalities. ${ }^{[10]}$ The interaction of water with amorphous solids lowers the glass transition temperature due to its universal plasticizing activity. The underlying molecular mechanisms may involve the binding of water molecules to the polar groups of the materials, that weaken the attracting forces between the polymer chains consequently it can increase the free volume [Figure 1]. Water molecules penetrate into the hydrophilic polymer matrix, the distance between the polymer chains will increase and thus more free volume will be available for molecular movements. ${ }^{[1]}$ In some cases, at higher water contents, the glass transition temperature of an excipient can decrease to room temperature and the material undergoes glassy-to-rubbery transition causing significant changes in their properties, such as increased reactivity and reduced chemical stability. ${ }^{[11]}$

Dlubeck et al. investigated plasticized polyamide 6 samples as a function of water content and temperature by positron annihilation lifetime spectroscopy (PALS). ${ }^{[12]}$ PALS is a unique method, which is able to measure free volume of polymers and other materials assuming spherical microholes (free volumes) in materials. It was found that the free volume of polyamide 6 decreased at low water contents compared to the initial (dry) free volume value and it was followed by an increase in the free volume at high water contents. This behaviour explained by the filling of the holes with water molecules at low water contents (initial stage of water sorption). At higher moisture contents water molecules weakened the hydrogen bonds between polymer chains and it led to the plasticization of the material and the increased the free volume. ${ }^{[12]}$

\section{MEASUREMENT TECHNIQUES}

\section{Water sorption experiments}

There are several methods that could characterize water-excipient interactions, such as inverse gas chromatography, ${ }^{[13]}$ NMR spectroscopy, ${ }^{[14]}$ dielectric relaxation spectroscopy, ${ }^{[15]}$ near infrared spectroscopy, ${ }^{[16]}$ etc. Water induced crystallization can be followed by X-ray diffraction measurements. ${ }^{[17]}$

A conventional method is the determination of the amount of sorbed water of materials as a function of environmental water activity (in practice, relative pressure, or relative humidity). Water

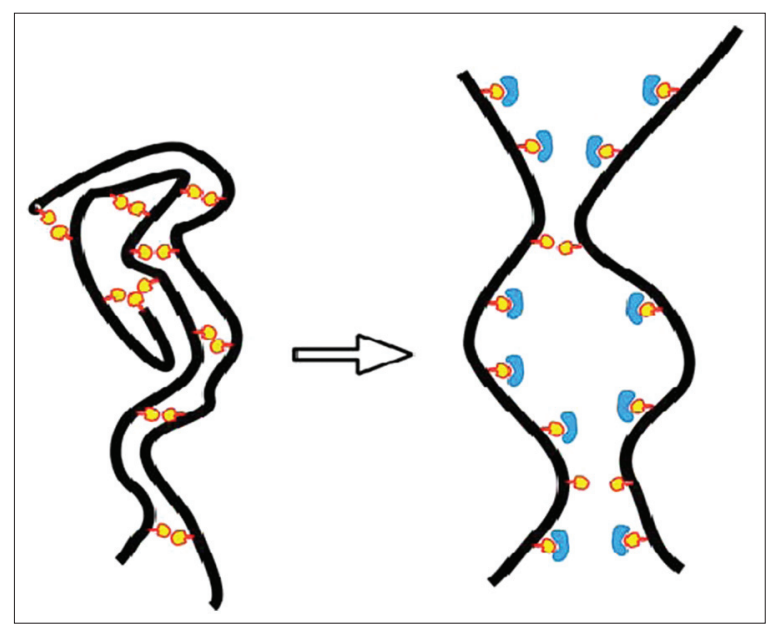

Figure 1: Possible effect of water sorption on the free volume of polymer materials 
sorption isotherms can be constructed by plotting the mass of sorbed water per unit mass of dry solid versus the relative pressure of water vapour $\left(\mathrm{P} / \mathrm{P}_{0}\right)$ or the relative humidity $\left(100\left(\mathrm{P} / \mathrm{P}_{0}\right)\right)$, where $\mathrm{P}_{0}$ is the vapour pressure of liquid water. ${ }^{[18]}$

Cellulose- and starch-based excipients are the most widely investigated materials by means of water sorption isotherms in the pharmaceutical field. The sorption-desorption curves of these excipients always show hysteresis, i.e., smaller amount of moisture leaves the material at a given relative humidity during desorption than it could be expected based on its sorption isotherm. The explanation of this phenomenon can be associated with the conformational change of the polymeric chains during water sorption, since water, penetrating into the structure of polymers, could alter the systems of inter- and intramolecular bonds. The shape of water sorption isotherms in the case of cellulose- and starch-based excipients was sigmoid, indicating the suitability of the use of equations developed for describing the physical adsorption of gases. ${ }^{[18]}$

Although the mechanisms of the water vapour absorption of amorphous materials greatly differ from the physical adsorption of gases on solid surfaces, due to the similarity of the shape of sorption isotherms, the well-known Brunauer-Emmett-Teller (BET) equation was successfully applied for the evaluation of the nature of the water vapour absorption. ${ }^{[19]}$

The Brunauer-Emmett-Teller (BET) equation:

$$
W=\frac{W_{M} C_{B}\left(\frac{P}{P_{0}}\right)}{\left[1-\left(\frac{P}{P_{0}}\right)\right]\left[1-\left(\frac{P}{P_{0}}\right)+C_{B}\left(\frac{P}{P_{0}}\right)\right]}
$$

Where $\mathrm{W}$ is the weight of sorbed water per unit mass of dry solid at a relative pressure of $\mathrm{P} / \mathrm{P}_{0} ; \mathrm{P}$ is the actual vapour pressure; $\mathrm{P}_{0}$ is the vapour pressure of liquid water; $\mathrm{W}_{\mathrm{M}}$ is the water content corresponding to one water molecule per sorption site; $\mathrm{C}_{\mathrm{B}}$ is a constant related to thermodynamical data of the system. The BET model assumes water sorption in two distinct thermodynamical states, where water molecules exist in a strongly bound state and as multilayer water similar to bulk water. Water sorption data of cellulose- and starch-based materials follow the BET equation only up to $0.3-0.4$ relative pressure. ${ }^{[18]}$ However this relative pressure range is sufficient to obtain the constants, $W_{M}$ and $C_{B}$, and to gain some information about water vapour sorption.

Zhang and Zografi investigated the water sorption isotherms of poly (vinyl pyrrolidone) (PVP) excipients of different molecular weights and they found that despite the significant differences between the values of calculated constant, $C_{B}$, the shape of the isotherms and the BET-parameter $\mathrm{W}_{\mathrm{M}}$ were very similar. The obtained results indicated that $\mathrm{W}_{\mathrm{M}}$ referred to the available hydration sites, whereas $C_{B}$ gave information about the overall free energy of absorption that could be varied with molecular weight of excipients. ${ }^{[19]}$
In the case of dried proteins it was observed, that its stability decreased if its water content exceed the monolayer value, because high water content allowed enhanced flexibility of macromolecules and increased the molecular mobility. On the other hand, if the water content of proteins was low enough it could also cause instability problems. Therefore, it seems to be favourable to keep water content of proteins at an intermediate level (near the monolayer value) to obtain satisfactory stability data. ${ }^{[20,21]}$

The GAB equation, developed by Guggenheim, Anderson and de Boer, could also provide the monolayer water sorption values $\left(\mathrm{W}_{\mathrm{M}}\right)$ and usually cover the water sorption data over the entire range of relative humidity, but minimum up to $80 \% \mathrm{RH}^{[22]}$ The GAB model assumes three state of water; i.e. free, tightly bound and intermediate water.

The GAB equation:

$$
W=\frac{W_{M} C_{G} K\left(\frac{P}{P_{0}}\right)}{\left[1-K\left(\frac{P}{P_{0}}\right)\right]\left[1-K\left(\frac{P}{P_{0}}\right)+C_{G}\left(\frac{P}{P_{0}}\right)\right]}
$$

where $\mathrm{W}$ and $\mathrm{W}_{\mathrm{M}}$ are the same as in the BET equation, $\mathrm{C}_{\mathrm{G}}$ and $\mathrm{K}$ are constants. The disadvantage of this equation is that the conformity of all constant to physical meanings is hardly possible. ${ }^{[18]}$

Roškar and Kmetec investigated the water sorption of several excipients (e.g. microcrystalline cellulose, crospovidone, copolividonum, magnesium stearate) using the BET and the GAB equations. They found that the experimental water sorption data were in good agreement with the BET model up to $55 \%$ $\mathrm{RH}$ and with the GAB model over the entire humidity range. The calculated $\mathrm{W}_{\mathrm{M}}$ values of water sorption of the excipients were similar in the case of both models, and the high values of these constants indicated that more than one layer of water molecules sorbed on some excipients. Particularly, in the case of crospovidone and copolividonum, where the $\mathrm{W}_{\mathrm{M}}$ values were high and these excipients were also very hygroscopic. ${ }^{[23]}$

\section{Differential scanning calorimetry}

In the case of polymeric materials the states of absorbed water are often characterized by differential scanning calorimetry (DSC). With DSC method three states of water was successfully identified by many experiments. States of water based on DSC measurements are the followings: (1) free water (FW), which undergoes similar phase transition near $0^{\circ} \mathrm{C}$ during heating (or freezing) as liquid water; (2) freezable bound water (FBW), which, due to the interaction with the polymer chains and/or capillary effects in the microholes of the polymer, undergoes phase transition at different temperature like liquid water; and (3) non-freezing (strongly) bound water (NBW), which does not undergo phase transition during the DSC experiments. The NBW is in direct contact with polar groups of polymers 
therefore it is unable to form ice crystals during freezing. The NBW is undetectable by the DSC method however its amount can be calculated if the total water content and the amount of FBW and FW are known. ${ }^{[7]}$

Hodge et al. investigating poly (vinyl alcohol) samples of different water contents found three states of water by DSC experiments. $\mathrm{Up}$ to $22 \%$ water content there was no detectable phase transition due to water molecules. Over $22 \%$ water content a sharp peak showed up with increasing intensity up to $28 \%$ water. At higher water contents a broad peak appeared with continuously increasing intensity. The sharp peak was associated with the phase transition of FBW. The sharpness of the peak was due to the interaction of water molecules with polymer chains and to capillary effects. In this state water molecules have a determined range of energetic states. The broad peak at higher water contents was due to the phase transition of free water. Subtracting the amount of FBW and FW from the total water content of a sample the amount of non-freezing water could be estimated using DSC measurements. Three states of water have also been observed by several researchers using differential scanning calorimetry investigating various polymers. ${ }^{[14,24-26]}$

Guan et al. investigated various polymers containing water using DSC and infrared spectroscopy. They found two endothermic DSC peaks in the case of each polymer associated with the freezing of freezable bound water (at lower temperatures) and freezing of free water (around $0^{\circ} \mathrm{C}$ ). The peaks at low temperatures were hardly observable in the case of some polymers therefore an isothermal treatment was carried out on these samples. At the isothermal treatment samples were kept a little above the transition temperature of its freezable bound water for a predetermined interval. During the time of the isothermal treatment more freezable bound water (FBW) was able to interact with the surrounding water molecules forming ice crystals than without the treatment since the structure and the amount of freezable bound water is not permanent and depends on the thermal history. In the case of one investigated polymer the researchers observed that the freezing temperature of FBW shifted to higher values after the isothermal treatment indicating that the small peak was due to the transition of the FBW. ${ }^{[27]}$

\section{Fourier transform infrared investigations}

The Fourier transform infrared (FTIR) spectroscopy is a useful method to investigate the states of water in polymers. Compared to the DSC and water sorption experiments it could offer substantially more information, since it provides an insight into the chemical structure of the investigated materials and after assignment of the infrared absorption bands to functional groups of polymers it is possible to determine the groups that are mainly affected by water molecules. ${ }^{[28]}$

Ping et al. investigated hydrophilic polymers of different water contents by DSC and FTIR methods. According to the DSC curves they found that the maximal amount of non-freezing bound water (NBW) of the polymers was dependent on its polar functional groups. For example, in the case of poly (vinyl pyrrolidone) four water molecules existed as $\mathrm{NBW}$ per $\mathrm{C}=\mathrm{O}$ groups indicating that one $\mathrm{C}=\mathrm{O}$ group can strongly interact with water molecules in two layers because one $\mathrm{C}=\mathrm{O}$ group have only two acceptor sites for hydrogen-bonding. ${ }^{[8]}$

For investigating water molecules of polymers the $3800-2900 \mathrm{~cm}^{-1}$ infrared region is the most suitable, because water molecules have strong absorption in this region due to its stretching vibrations. However the polymer molecules often also have infrared (IR) absorption in this region, therefore difference spectra could be more informative for water molecules as the original ones. To construct the difference spectra the normalized spectrum of dry sample has to be subtracted from the normalized spectra of the wetted samples. The shape of the difference spectra in the $3800-$ $2900 \mathrm{~cm}^{-1}$ region will be characteristic of the water molecules in the polymer. Differences between the thicknesses of samples could be taken into account with spectra normalization.

Ping et al. investigated poly (vinyl alcohol) (PVA) samples of different water contents by FTIR spectroscopy and evaluated the difference spectra. They found that the $3800-2900 \mathrm{~cm}^{-1}$ region markedly differed from the spectrum of liquid water, because system of hydrogen-bonds of water molecules changed due to their interaction with the polymer. The band intensities in this region increased with increasing water content of the samples. In this region two distinct, overlapping bands were seen at nearly fixed wavenumber values but with different intensities according to the actual water contents of the samples. The peak at lower wavenumber, $3280 \mathrm{~cm}^{-1}$, was more intense up to $20 \%$ water content but at higher water contents the peak at $3400 \mathrm{~cm}^{-1}$ was more pronounced. ${ }^{[8]}$ Based on this observation, water molecules of the polymer could be classified into two main categories; strongly hydrogen-bonded and water molecules of bulk (free) water. The strongly hydrogen-bonded water molecules appeared in the lower wavenumber region, because the interactions with polar groups of the polymer caused shift in the infrared absorption of water molecules to the lower wavenumbers. The changes in the relative intensities of the two distinct peaks of water molecules indicated that the ratio of strongly hydrogen-bonded water molecules moved to free water as the water content increased due to the saturation of strong binding sites of PVA.

The broad band of stretching vibrations of water in the difference spectra can be further evaluated by deconvoluting (other words decomposing) this region into components using curve fitting algorithms. After deconvolution the components could be assigned to water molecules of different strength of hydrogen-bonds, because in the reality water molecules exist in many different states [Figure 2]. Lasagabaster et al. investigated stretching vibrations of water molecules absorbed by polypropylene (PP)/ethylenealcohol vinyl (EVOH) films based on difference spectra. They deconvoluted the water stretching region into four components assume Gaussian-shape of the individual components. According to the wavenumber values of the components they classified water molecules into categories: non hydrogen-bonded, weakly 


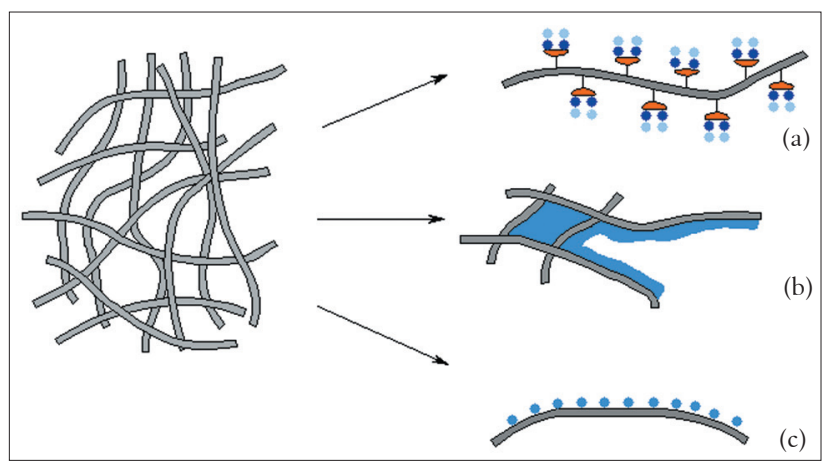

Figure 2: Possible association of water with the polymer molecules; (a) water molecules strongly attached to the polar groups of the polymer chains by hydrogen-bonds in two layers; (b) microscopic voids inside the polymer filled with excess water; (c) individual water molecules attached to the non-polar region of the polymer by weak interaction forces

hydrogen-bonded, medium hydrogen bonded and strongly hydrogen-bonded. ${ }^{[29]}$ According to other experiments, there exist some water molecules even in the liquid water, which are not hydrogen-bonded based on IR spectral data. ${ }^{[30]}$ The medium hydrogen-bonded water molecules were attributed to the second hydration layer in the case of $\mathrm{PP} / \mathrm{EVOH}$ films and the strongly hydrogen bonded molecules (deconvoluted component at the lowest wavenumber) to water molecules attached directly by polymer chains. ${ }^{[29]}$ Consequently, the band deconvolution could provide abundant information about water molecules hydrogenbonded of different strengths, however the interpretation of data is more difficult compared to the DSC results due to the complex nature of an infrared spectrum, which is not detailed here.

Other authors investigated water absorption of epoxy matrices and they also deconvoluted the stretching vibrations of water into four components to obtain the individual spectra of water molecules of different hydrogen-bonds. ${ }^{[31]}$ They found that plotting the intensity of the components versus relative humidity the ratio of weakly hydrogen-bonded molecules increased with increasing water contents. This result were in accordance with the expectation that with increasing water content the ratio of weakly hydrogen bonded water molecules increase compared to the strongly hydrogen-bonded ones due to the saturation of the polar groups of the epoxy samples.

Olsson and Salmen investigated water absorption of cellulose based-materials. They recorded IR spectra of samples kept at $80 \% \mathrm{RH}$ for each $10 \% \mathrm{RH}$ for 60 minutes to water absorption. Two distinct peaks, at 3600 and $3200 \mathrm{~cm}^{-1}$ were observed on the difference spectra of the samples of different water contents. Surprisingly, the same relative peak heights were found in the case of each sample suggesting that weakly hydrogen-bonded and strongly hydrogen-bonded water molecules were present in similar amount even at low water contents. This result is contradictory to the model based on DSC results, where, in the case of hydrophilic polymers, the presence of strongly bounded water molecules is supposed at low water contents and freezable bound water appears only with increasing water contents which is followed by the free

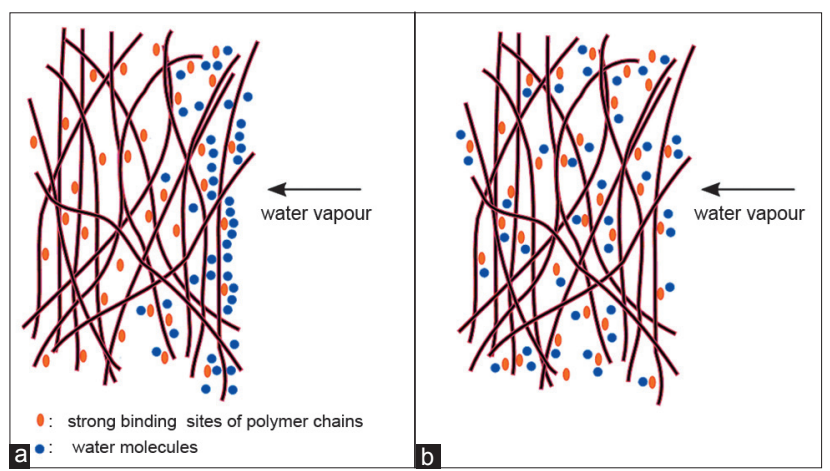

Figure 3: Possible mechanisms of water vapour absorption into hydrophilic amorphous polymers at the initial stage of water absorption; (a) water attached by clusters of molecules to polymer chains simultaneously absorbing to binding sites of different strengths; (b) water molecules first bounded to the strongest binding sites of the polymer chains

water. In the FT-IR experiments the evolving of the two peaks with same relative heights indicated water absorption by clusters, where water clusters adhere simultaneously to all binding sites of the polymer even at low water contents ${ }^{[32]}$ [Figure 3]. Similar discrepancies were found by other authors, too. ${ }^{[29]}$ There is a lack of a proper physical model that is able to explain all findings observed by water sorption method, DSC and FTIR spectroscopy.

\section{EFFECTS OF WATER ON THE PROPERTIES OF DOSAGE FORMS}

It is well-known that moisture sorption of solid dosage forms can affect its chemical, physico-chemical and microbial stability. One of the most important issues is the chemical stability of the active pharmaceutical ingredient (API) of the final dosage form. Absorbed water could initiate the chemical degradation of the API by different mechanisms. The amorphous state is of impact in this field, because an amorphous solid is able to absorb large amount of water to its bulk structure, and usually the degradation rate of an API is dependent on the water content. In addition to this, several researchers showed that the reactivity of an amorphous substance is greater than its crystalline equivalent under identical conditions. ${ }^{[33]}$

Shalaev and Zografi detailed four possible mechanisms that can be responsible for the degradation activity of water: ${ }^{[33]}$ (1) water could be a direct participant in several degradation reactions of drugs (e.g., hydrolytic reactions), therefore its concentration could largely affect the stability of the final product; (2) it could be a product of specific reactions, in this case its increasing amount could inhibit the progress of these reactions; (3) the water dissolved in the amorphous matrix could be also the medium of reactions by changing the polarity and the reactivity of the surrounding molecules; (4) finally, water acts as a plasticizer which could significantly reduce the glass transition temperature, and increase the molecular mobility and the free volume of the system. On the other hand, effects of water are not independent therefore it is difficult to distinguish its role as reactant, medium of reactions, and plasticizer in degradation processes. ${ }^{[34]}$ 
Lai et al. investigated the effect of sorbed water and glass transition temperature on deamidation of an asparaginecontaining hexapeptide lyophilized with PVP. Glycerol was added to the mixture in different extents to modifiy the glass transition temperature of the system containing a given amount of water. Therefore, it was possible to make samples of similar water contents but different glass transition temperatures $\left(\mathrm{T}_{\mathrm{g}}\right)$ and samples of similar $\mathrm{T}_{\mathrm{g}}$ values but different water contents. The stability testing was performed at $50^{\circ} \mathrm{C}$ and due to the different plasticization of the lyophilised mixtures there were samples in the glassy and also in the rubbery state during the stability testing. Decreased stability was observed with increased glycerol but similar water content indicating that reduced $\mathrm{T}_{\mathrm{g}}$ adversely affected the chemical stability of the peptide even if the water content was not significantly different. It was also showed that the deamidation of the investigated peptide samples was differently affected by water in the glassy and in the rubbery state. Along with increasing water content, in the glassy state of similar $\mathrm{T}_{\mathrm{g}}$ values the degradation rate constants of samples also increased, while in the case of samples in the rubbery state only the $\mathrm{T}_{\mathrm{g}}$ determined the rate constant. ${ }^{[34]}$ Consequently, water induced degradation mechanisms in pharmaceuticals could be explained by the role of water as direct participant in chemical reactions but also by its plasticizing effect that can change the properties of the entire complex delivery system (e.g., molecular mobility, free volume, polymer chain conformation, etc.).

The molecular mobility often plays an important role in the degradation rate. Along with the glassy-to-rubbery transition of an amorphous material the molecular mobility of the system change therefore the $T_{g}$ value of the material could play an important role from the point of chemical stability. In fact, both degradation of various small molecules and aggregation of proteins showed change in the slope of the temperature dependence of the degradation rate around $\mathrm{T}_{\mathrm{g}}$, indicating a nonArrhenius like behaviour in this temperature range. ${ }^{[1]]}$

Besides the chemical stability, residual water could affect a range of parameters that are important in view of quality, safety, and efficacy of pharmaceuticals. Moisture content of starch excipients has a great importance during tablet compaction. Optimum water content could enhance both the plastic deformation of the particles and the compressibility. ${ }^{[35]}$

Mihranyan et al. examined the influence of the properties of cellulose powders on the moisture-induced degradation of acetylsalicylic acid (ASA). Different grades of cellulose powders were used of various crystallinity, specific surface area, and moisture absorption. The crystallinity and water content of the excipients had a great influence on the degradation rate, the cellulose powder of the lowest crystallinity (and consequently of highest moisture absorption and content) provided the highest stability of the drug. On the other hand, highest instability was observed in the case of cellulose sample of highest crystallinity and specific surface area but of low hygroscopicity, despite the hydrolytic mechanism of ASA degradation. The authors concluded that, the location and availability of sorbed moisture was more important than the overall water content of the system. ${ }^{[36]}$

Water content of amorphous or partially amorphous hydrophilic polymers has a great importance during tableting processes, because the properties of these pharmaceutical excipients could markedly change due to the plasticizing effect of water. Minimum water content is essential for direct compaction of starch-based materials, because water could provide good compressibility and plastic deformation of particles. Steendam et al. investigated the behaviour of amylodextrin in the $0.07<\mathrm{x}_{\mathrm{w}}<0.4$ moisture fraction $\left(\mathrm{x}_{\mathrm{w}}\right)$ range. The $\mathrm{T}_{\mathrm{g}}$ of amylodextrin-water blends was equal to room temperature at 0.19 moisture fraction determined by DSC. Plasticizing effects of water on amylodextrin compacts was evaluated by compressive stress-strain experiments. The elastic modulus of the system steeply decreased between $\mathrm{x}_{\mathrm{w}}=0.17$ and 0.23 that was in accordance with the glassy-to-rubbery transition of the material of $0.19 \mathrm{x}_{\mathrm{w}}$ at room temperature. The compressibility of powder was also markedly changed in the rubbery state. ${ }^{[37]}$

Absorbed water could change the free volumes of moisture sensitive excipients in dosage forms.

The effect of free volume changes during storage at high relative humidity values on drug release characteristics was studied on tablets. ${ }^{[38,39]}$ The release rate of theophylline, a crystalline non-hygroscopic drug from wet-granulated tablets containing amorphous PVP as binder was greatly affected by storage conditions, showed by Zelkó and Süvegh. Storage of the excipient at $65 \% \mathrm{RH}$ for 30 days induced a glassy-to-rubbery transition and according to PALS measurements this relative humidity value and the consequent water sorption was high enough to the phase transition. ${ }^{[38,40]}$ The structural changes of the binder began at $65 \% \mathrm{RH}$ also affected the properties of the tablets and the release of the drug was slightly retarded. At $75 \% \mathrm{RH}$, where the complete transition of the binder occurred, the mean release time of theophylline was reduced to the half of the original value due to the large scale changes of the binder molecules. ${ }^{[38,41]}$

Polymeric excipient molecules with polar groups are able to form hydrogen bonds with drug molecules during tablet preparation and dissolution. Szente et al. investigated the interaction of metronidazole with carbomer excipient (Carbopol ${ }^{\circledR}$ ) using FTIR spectroscopy and $a b$ initio computational calculations. Based on these calculations it could be expected that a special arrangement could occur between Carbopol ${ }^{\circledR}$ and metronidazole with relatively high hydrogen-bonding energy. It was observed that after two-week of storage of the tablets at $75 \% \mathrm{RH}$ the dissolution profile was markedly changed. The authors concluded that absorbed water molecules were able to break the bonds between metronidazole and excipient molecules inducing faster release of drug molecules. ${ }^{[39]}$

In the case of protein-containing inhalation powders the relative humidity of storage conditions has also a great importance. The storage environment of inhalation powders could affect the 
physical stability of the dosage form and the chemical stability of the API, as well. Maa et al. investigated the physical stability of protein preparations blended with lactose carrier and they found that it was less affected after exposure to different relative humidity circumstances. However, the protein preparations were hygroscopic and its chemical stability was largely affected by storage conditions. It was found that the aggregation of the anti$\mathrm{IgE}$ antibody preparation was increased with increasing relative humidity and at higher temperatures the effect of environmental relative humidity was more pronounced on the aggregation rate than at lower temperatures. ${ }^{[42]}$ In the case of inhalation powders the storage conditions must be well-defined, since even if the carrier excipient is not hygroscopic and the physical stability of the product is maintained, the drug molecules could decompose induced by water if the active part of the formulation is able to absorb moisture.

\section{REFERENCES}

1. Abiad MG, Carvajal MT, Campanella OH. A review on methods and theories to describe the glass transition phenomenon: Applications in food and pharmaceutical products. Food Eng Rev 2009;1:105-32.

2. Hancock BC, Zografi G. Characteristics and significance of the amorphous state in pharmaceutical systems. J Pharm Sci 1997;86:1-12.

3. Yu L. Amorphous pharmaceutical solids: Preparation, characterization and stabilization. Adv Drug Deliver Rev 2001;48:27-42.

4. Craig DQ, Royall PG, Kett VL, Hopton ML. The relevance of the amorphous state to pharmaceutical dosage forms: Glassy drugs and freeze dried systems. Int J Pharm 1999;179:179-207.

5. Jadhav NR, Gaikwad VL, Nair KJ, Kadam HM. Glass transition temperature: Basics and application in pharmaceutical sector. Asian J Pharm 2009;3:82-9.

6. Ottenhof MA, MacNaughtan W, Farhat IA. FTIR study of state and phase transitions of low moisture sucrose and lactose. Carbohyd Res 2003;338:2195-202.

7. Hodge RM, Edward GH, GP Simon. Water absorption and states of water in semicrystalline poly(vinyl alcohol) films. Polymer 1996;37:1371-6.

8. Ping ZH, Nguyen QT, Chen SM, Zhou JQ, Ding YD. States of water in different hydrophilic polymers - DSC and FTIR studies. Polymer 2001;42:8461-7.

9. Hancock BC, Shamblin SL. Water vapour sorption by pharmaceutical sugars. Pharm Sci Technol Today 1998;1:345-51.

10. Thibert R, Hancock BC. Direct visualization of superdisintegrant hydration using environmental scanning electron microscopy. J Pharm Sci 1996;85:1255-8.

11. Yoshioka S, Aso Y. Correlations between molecular mobility and chemical stability during storage of amorphous pharmaceuticals. J Pharm Sci 2007;96:960-81.

12. Dlubek G, Redmann F, Krause-Rehberg R. Humidity-induced plasticization and antiplasticization of polyamide 6: A Positron lifetime study of the local free volume. J Appl Polym Sci 2002;84:244-55.

13. Surana R, Randall L, Pyne A, Vemuri NM, Suryanarayanan R. Determination of glass transition temperature and in situ study of the plasticizing effect of water by inverse gas chromatography. Pharm Res 2003;20:1647-54.

14. Liu WG, Yao KD. What causes the unfrozen water in polymers: Hydrogen bonds between water and polymer chains? Polymer 2001;42:3943-7.
15. Motwania T, Lanagan M, Anantheswarana RC. State of water in starch-water systems in the gelatinization temperature range as investigated using dielectric relaxation spectroscopy. Carbohyd Polym 2012;87:24-31.

16. Ohtake N, Yonemochi E, Terada K. Characterization of the molecular state of water present in pharmaceutical additives by NIR spectroscopy. Asian J Pharm Sci 2006;1:43-6.

17. Tong P, Zografi G. Effects of water vapor absorption on the physical and chemical stability of amorphous sodium indomethacin. AAPS PharmSciTech 2004;5:9-16.

18. Zografi G, Kontny MJ. The interactions of water with celluloseand starch-derived pharmaceutical excipients. Pharm Res 1986;3:187-94.

19. Zhang J, Zografi G. The Relationship between "BET"- and "Free Volume"-derived parameters for water vapor absorption into amorphous solids. J Pharm Sci 2000;89:1063-72.

20. Costantino HR, Curley JG, Hsu CC. Determining the water sorption monolayer of lyophilized pharmaceutical proteins. J Pharm Sci 1997;86:1390-3.

21. Chang LL, Shepherd D, Sun J, Tang X, Pikal MJ. Effect of sorbitol and residual moisture on the stability of lyophilized antibodies: Implications for the mechanism of protein stabilization in the solid state. J Pharm Sci 2005;94:1445-55.

22. Timmermann EO. Multilayer sorption parameters: BET or GAB values? Colloid Surface A. 2003;220:235-60.

23. Roškar R, Kmetec V. Evaluation of the moisture sorption behaviour of several excipients by BET, GAB and microcalorimetric approaches. Chem Pharm Bull 2005;53:662-5.

24. Baba T, Sakamoto R, Shibukawa M, Oguma K. Solute retention and the states of water in polyethylene glycol and poly(vinyl alcohol) gels. J Chromatogr A 2004;1040:45-51.

25. Maeda T, Yamamoto K, Aoyagi T. Importance of bound water in hydration-dehydration behaviour of hydroxylated poly $(\mathrm{N}-$ isopropylacrylamide). J Colloid Interf Sci 2006;302:467-74.

26. Ruiz J, Mantecón A, Cádiz V. States of water in Poly(vinyl alcohol) derivative hydrogels. J Polym Sci Pol Phys 2003;41:1462-7.

27. Guan L, Xu H, Huang D. The investigation on states of water in different hydrophilic polymers by DSC and FTIR. J Polym Res 2010;18:681-9.

28. Haxaire K, Maréchal $Y$, Milas M, Rinaudo M. Hydration of polysaccharide hyaluronan observed by IR Spectrometry. I. Preliminary experiments and band assignments. Biopolymers 2003;72:10-20.

29. Lasagabaster A, Abad MJ, Barral L, Ares A. FTIR study on the nature of water sorbed in polypropylene (PP)/ethylene alcohol vinyl (EVOH) films. Eur Polym J 2006;42:3121-32.

30. Maréchal $Y$. Observing the water molecule in macromolecules and aqueous media using infrared spectrometry. J Mol Struct 2003;648:27-47.

31. Cotugno S, Larobina D, Mensitieri G, Musto P, Ragosta G. A novel spectroscopic approach to investigate transport processes in polymers: The case of water-epoxy system. Polymer 2001;42:6431-8.

32. Olsson AM, Salmén L. The association of water to cellulose and hemicellulose in paper examined by FTIR spectroscopy. CarbohyDr. Res 2004;339:813-8.

33. Shalaev EY, Zografi G. How does residual water affect the solidstate degradation of drugs in the amorphous state? J Pharm Sci 1996;85:1137-41.

34. Lai MC, Hageman MJ, Schowen RL, Borchardt RT, Laird BB, Topp EM. Chemical stability of peptides in polymers. 2. Discriminating between solvent and plasticizing effects of water on peptide deamidation in poly(vinylpyrrolidone). J Pharm Sci 1999;88:1081-9. 
35. Yoon HS, Kweon DK, Lim ST. Effects of drying process for amorphous waxy maize starch on theophylline release from starch-based tablets. J Appl Polym Sci 2007;105:1908-13.

36. Mihranyana A, Strømmeb M, Ek R. Influence of cellulose powder structure on moisture-induced degradation of acetylsalicylic acid. Eur J Pharm Sci 2006;27:220-5.

37. Steendam R, Frijlink HW, Lerk CF. Plasticisation of amylodextrin by moisture. Consequences for compaction behaviour and tablet properties. Eur J Pharm Sci 2001;14:245-54.

38. Zelkó R, Süvegh K. Correlation between the release characteristics of theophylline and the free volume of polyvinylpyrrolidone. Eur J Pharm Sci 2005;24:351-4.

39. Szente V, Baska F, Zelkó R, Süvegh K. Prediction of the drug release stability of different polymeric matrix tablets containing metronidazole. J Pharm Biomed Anal 2011;54:730-4.

40. Süvegh K, Zelkó R. Physical aging of poly(vinylpyrrolidone) under different humidity conditions. Macromolecules 2002;35:795-800.

41. Zelkó R, Orbán A, Süvegh K. Tracking of the physical ageing of amorphous pharmaceutical polymeric excipients by positron annihilation spectroscopy. J Pharm Biomed Anal 2006;40: 249-54.

42. Maa YF, Nguyen PA, Andya JD, Dasovich N, Sweeney TD, Shire SJ, et al. Effect of spray drying and subsequent processing conditions on residual moisture content and physical/biochemical stability of protein inhalation powders. Pharm Res 1998;15: 768-75.

How to cite this article: Szakonyi G, Zelkó R. The effect of water on the solid state characteristics of pharmaceutical excipients: Molecular mechanisms, measurement techniques, and quality aspects of final dosage form. Int J Pharma Investig 2012;2:18-25.

Source of Support: Nil. Conflict of Interest: None declared.

\section{Author Help: Online submission of the manuscripts}

Articles can be submitted online from http://www.journalonweb.com. For online submission, the articles should be prepared in two files (first page file and article file). Images should be submitted separately.

1) First Page File:

Prepare the title page, covering letter, acknowledgement etc. using a word processor program. All information related to your identity should be included here. Use text/rtf/doc/pdf files. Do not zip the files.

2) Article File:

The main text of the article, beginning with the Abstract to References (including tables) should be in this file. Do not include any information (such as acknowledgement, your names in page headers etc.) in this file. Use text/rtf/doc/pdf files. Do not zip the files. Limit the file size to $1024 \mathrm{~kb}$. Do not incorporate images in the file. If file size is large, graphs can be submitted separately as images, without their being incorporated in the article file. This will reduce the size of the file.

3) Images:

Submit good quality color images. Each image should be less than $\mathbf{4 0 9 6} \mathbf{~ k b ~ ( 4 ~ M B ) ~ i n ~ s i z e . ~ T h e ~ s i z e ~ o f ~ t h e ~ i m a g e ~ c a n ~ b e ~ r e d u c e d ~ b y ~ d e c r e a s i n g ~}$ the actual height and width of the images (keep up to about 6 inches and up to about $1800 \times 1200$ pixels). JPEG is the most suitable file format. The image quality should be good enough to judge the scientific value of the image. For the purpose of printing, always retain a good quality, high resolution image. This high resolution image should be sent to the editorial office at the time of sending a revised article.

4) Legends:

Legends for the figures/images should be included at the end of the article file. 